\title{
Transport of Flight Critical Data over Internet Protocol
}

\author{
Vijay Ragothaman, Fariha Baloch* and Dr. Ravi Pendse
}

Department of Electrical and Computer Engineering, College of Engineering

\begin{abstract}
The aviation industry forms an important component of the nation's critical infrastructure. By considering the issues involved in protecting the operational aspects of the aviation industry, in conjunction with the introduction of Internet access within an airplane, the authors present the possibility of providing an additional path for the transport of critical content between and airplane and ground stations
\end{abstract}

\section{Introduction}

The National Airspace System (NAS) comprises of a complex network of interconnected systems in addition to the people who operate, maintain and use the systems and detailed procedures and certifications. One of the main components of this infrastructure is the Air Traffic Controller (ATC) since it is a hub for all the communications between all airplanes and is responsible for safe landing and take-off of the planes. In this research authors have build up on previous research where using IP was suggested as a method of communicating between pilot and ATC instead of using regular Very High Frequency (VHF) band. This approach is also useful if VHF band gets congested due to high number of traffic flows. ATCs and pilots have had to rely on third party High Frequency (HF) communication relays for exchanging voice messages. With the introduction of Controller Pilot Data Link Communication (CPDLC), which provides for direct exchange of text-based messages between the ATC and the pilot, the need for a third party relay is eliminated. Results from previous research work indicate that the presence of an Internet Protocol (IP) network between the aircraft and ground station presents the possibility of secure transfer of flight data and cockpit voice in real-time to ground stations. This provides an opportunity to route the CPDLC traffic through the IP link to the ground station, rather than share a HF link with several other aircrafts in the vicinity.

For the current work, the authors propose a mechanism to monitor the Quality of Service (QoS) of the CPDLC traffic using IP connectivity and reroute the communication via the regular HF link in case of QoS deterioration.

In order to reduce congestion over the VHF links due to voice communication between ATC and aircrafts, data link communication is being implemented as an alternative. ADLS (Aeronautical Data Link) has CPDLC as its leading application. CPDLC provides the capability to display air traffic communications exchanged between the controller and the flight deck, thereby reducing the dependence on voice communications. Some of the benefits of using distributed communications methods (voice and data) are as follows:

- Increased flight efficiency reflected by less time and fewer miles flown in sector will improve on-time arrivals. CPDLC reduces frequency congestion, therefore allows more timely and efficient delivery of clearances.

- Increased airspace capacity reflected by increased sector traffic throughput and reduced delay. Increased capacity enabled by controller productivity gains associated with automating messages will reduce arrival delays.

- Enhanced safety reflected by decreased operational errors and increased communications accuracy. [1]

\section{QoS Mechanism}

There are inherent disadvantages in using the VHF link for controller-pilot communications and the previous section presented some of them. The presence of an IP network between aircraft and ground stations leads to the possibility of using the IP link for air-ground communications. The communication between the pilot and ATC is very critical and hence security is an important aspect that should be considered. The VHF link used for controller-pilot communications is a shared medium and this can lead to disruptions in 
communication with an increase in the number of aircrafts in a given airspace, which is undesirable.

The above observations present the possibility of routing the Controller-Pilot Communication (CPC) traffic via the IP link. This can be achieved in three different ways, namely, voice only, data only, both voice and data. The implementation of either of these three can be done as in [2]

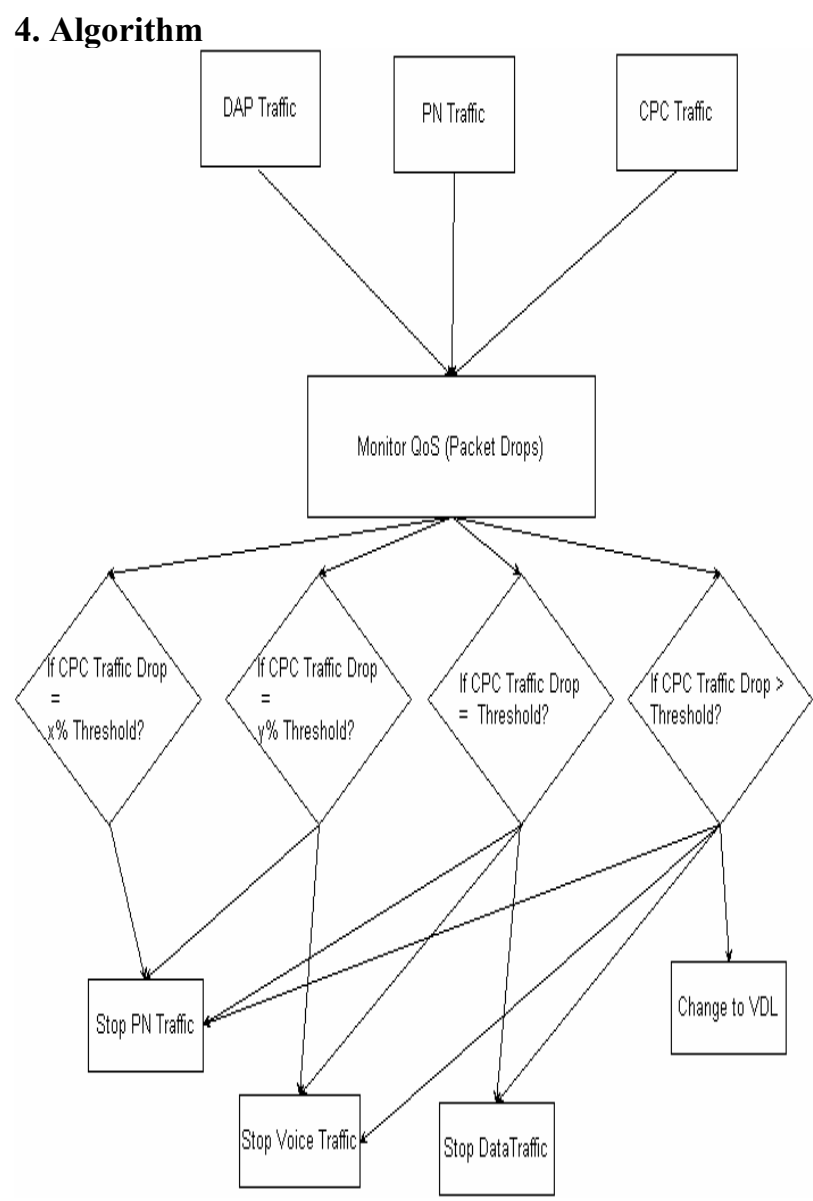

Figure 1: Flow Control for QoS Mechanism

Controller-pilot communication is not a continuous stream of voice or data messages. Hence, this should not pose a problem as far as bandwidth is concerned. However, the critical nature of this traffic requires continuous monitoring in case of failure or congestion in the IP link or loss of voice/data integrity. In view of this the authors propose a mechanism described by Figure 1 to monitor the Quality of Service of the controller-pilot communication traffic. In case of quality deterioration, leading to loss of data, the communication is rerouted through the VHF data link. The authors define Threshold as the percentage of packet drop at which the controller-pilot communication data is not understood at either end.
As seen from Figure 1, the Quality of Service for the different traffic streams is monitored in terms of packet drops. Additionally, voice quality is measure using PSQM scores. The following steps describe the rest of the flow.

- If the CPC packet drops reaches $\mathrm{x} \%$ of the threshold, the Passenger Network traffic is shut down until the packet drops falls below $\mathrm{x}$.

- If the CPC packet drops reaches y $\%(x<y)$ of threshold, the Voice traffic is shut down until the packet drops fall below y.

- If the CPC packet drops reach the threshold, the Data traffic is shut down until the packet drops fall below the threshold.

- If the CPC packet drops exceed the threshold, the CPC traffic is rerouted through the VHF links.

- Once the IP link is restored to normality, say congestion is cleared, the CPC traffic can be restored via the IP link.

\section{Conclusion and Future Work}

The implementation of the QoS mechanism for reverting to VHF links is a work in progress. Based on the DAP simulations that were performed, it was concluded that the quality of voice was found to be at acceptable levels due to the constraints placed by the satellite link in terms of bandwidth and delay. The DAP results in [2] reveal the fact that data traffic requires less than $10 \mathrm{kbits} / \mathrm{sec}$ of bandwidth. This is indicative that Controller-Pilot communication by means of data traffic seems to be a better option, but needs to be verified by simulations to test its integrity. As part of future work, the authors will be looking to incorporate security features into this framework along with PEP to improve TCP throughput over satellite links. The Communications part of CNS has been discussed in this paper. The Navigation and Surveillance areas were outside the scope of this paper, but can be looked into as they form a critical part of the aircraft network. It will also be interesting to see the issues involved in establishing secure communication between two aircrafts without the need for a ground station.

\section{Reference:}

[1] National Airspace System Operational Evolution Plan Version 7.0, The Federal Aviation Administration, http://www.faa.gov/programs/oep/v7/smart $\% 20$ sheets/policy \%20datalink.htm

[2] Ragothaman, Thanthry, Bhagavathula and Pendse, 2004, IP Connectivity and DAP, Wichita State University, Digital Avionics System Conference. 